\title{
REPRESENTATION THEOREMS FOR POSITIVE SOLUTIONS OF PARABOLIC EQUATIONS
}

\author{
YEHUDA PINCHOVER
}

(Communicated by Walter D. Littman)

\begin{abstract}
We determine all the minimal positive solutions of the parabolic equation $L u=0$ in $\mathbf{R}^{n} \times \mathbf{R}_{-}$, where $L$ has time independent coefficients or $L$ has periodic coefficients in $x_{1}, \ldots, x_{n}$ and $t$.
\end{abstract}

0. Introduction. Consider the second order parabolic equation

$$
L u=u_{t}+P u=0
$$

in a domain $D \subseteq \mathbf{R}^{n} \times \mathbf{R}$. We are interested to find an integral representation theorem for all nonnegative solutions of $(0.1)$ in $D$.

Widder [10] proved that $u$ is a nonnegative solution of the heat equation

$$
u_{t}-\frac{\sigma^{2}}{2} \Delta u=0 \text { in } \mathbf{R}^{n} \times \mathbf{R}_{+},
$$

iff

$$
u(x, t)=\int_{\mathbf{R}^{n}}\left(2 \pi \sigma^{2} t\right)^{-n / 2} \exp \left[\left(2 \sigma^{2} t\right)^{-1}|x-\eta|^{2}\right] d \mu(\eta),
$$

where $\mu$ is a Borel measure on $\mathbf{R}^{n}$.

This result was extended by Friedman [3] and Aronson [2] to general second order parabolic equations.

Using Appell transform one can show that a nonnegative solution $u(x, t)$ of the heat equation (0.2) in $\mathbf{R}^{n} \times \mathbf{R}_{-}$or in $\mathbf{R}^{n} \times \mathbf{R}$ is given by

$$
u(x, t)=\int_{\mathbf{R}^{n}} \exp \left(\gamma \cdot x+\frac{\sigma^{2}|\gamma|^{2} t}{2}\right) d \mu(\gamma) .
$$

In other words, every nonnegative solution of $(0.2)$ in $\mathbf{R}^{n} \times \mathbf{R}_{-}$is a convex combination of positive exponential solutions.

In this paper we generalize the last result to parabolic equations with periodic coefficients. The proofs of our results are adaptations of the proofs given by $\mathrm{S}$. Agmon and by the author for the elliptic case ([1]; see also [4]). We would like to remark that the results of this paper were first announced in [8].

ACKNOWLEDGEMENTS. This paper is based on one part of the author's Ph.D. Thesis that was completed in 1986 at the Hebrew University of Jerusalem [9]. I wish to express my deep gratitude to my thesis advisor, Professor Shmuel Agmon for the encouragement, support and help he gave me.

Received by the editors November 6, 1987.

1980 Mathematics Subject Classification (1985 Revision). Primary 35C15, 35K10; Secondary 35B05.

Key words and phrases. Appell transform, cap, Cauchy problem, exponential solution, extreme point, heat equation. 
1. Preliminaries. We shall consider a second order parabolic operator $L$ acting on functions $u$ in a domain $D \subseteq \mathbf{R}^{n} \times \mathbf{R}, n \geq 2$. We shall assume that $L$ is of the form:

$$
L u=u_{t}-\sum_{i, j=1}^{n} a_{i j}(x, t) \partial_{i} \partial_{j} u+\sum_{i=1}^{n} b_{i}(x, t) \partial_{i} u+c(x, t) u,
$$

$\partial_{i}=\partial / \partial x_{i}, \quad x=\left(x_{1}, \ldots, x_{n}\right)$. We assume that the coefficients of $L$ are real measurable functions and that

$$
\mu^{-1} \sum_{i=1}^{n} \xi_{i}^{2} \leq \sum_{i, j=1}^{n} a_{i j}(x, t) \xi_{i} \xi_{j} \leq \mu \sum_{i=1}^{n} \xi_{i}^{2}
$$

for all $(x, t) \in D$ and $\xi \in \mathbf{R}^{n}, \mu$ is a positive constant. We assume further that

$$
a_{i j} \in C(D) \quad \text { and } \quad \sum_{i=1}^{n}\left|b_{i}(x, t)\right|+|c(x, t)| \leq \mu \quad \text { for all }(x, t) \in D .
$$

DEFINITION 1.1. $u(x, t)$ is a (strong) solution of the equation $L u=0$ in $D$, if $u \in W_{\text {loc }}^{2 ; 1, n+1}(D)$ and $u$ satisfies $L u=0$ a.e. in $D$.

Denote

$$
Q=Q\left(x_{0}, t_{0}, R, \theta\right)=\left\{(x, t) \in \mathbf{R}^{n} \times \mathbf{R}|| x-\left.x_{0}\right|_{\infty}<R, t \in\left(t_{0}, t_{0}+\theta R^{2}\right)\right\} .
$$

From Sobolev's imbedding theorem it follows that if $u \in W^{2 ; 1, n+1}(Q)$ then there exists $v$, a continuous function on $\bar{Q}$ s.t. $v=u$ a.e. in $Q$. Therefore from now on we shall assume that all solutions of $L u=0$ in $D$ are continuous functions.

REMARK 1.2. The mapping $u(x, t) \rightarrow \tilde{u}(x, t)=e^{-\mu t} u(x, t)$ maps a nonnegative solution of the equation $L u=0$ in $D$ to a nonnegative solution of the equation $\tilde{L} \tilde{u}=L \tilde{u}+\mu \tilde{u}=0$ in $D$. Notice that the zero order term of $\widetilde{L}$ is $\tilde{c}(x, t)=c(x, t)+\mu$, and that $0 \leq \tilde{c}(x, t) \leq 2 \mu$. Since we are interested in representation theorems for the set of all nonnegative solutions of $L u=0$ in $D$, we can assume that $c(x, t)$ is a nonnegative bounded function in $D$.

Krylov and Safanov [5] proved the following Harnack inequality.

THEOREM 1.3. Let $u$ be a nonnegative solution of the equation $L u=0$ in $Q=Q\left(x_{0}, t_{0}, R, \theta\right)$, and assume that $\theta>1$ and $R \leq 2$. Then

$$
u\left(x_{0}, t_{0}+R^{2}\right) \leq C u\left(x, t_{0}+\theta R^{2}\right)
$$

for every $\left|x-x_{0}\right|_{\infty} \leq R / 2$, where $C=C(\theta, \mu, n)$. Moreover $C$ varies within bounded bounds for all $\theta>1$ s.t. $0<\varepsilon \leq(\theta-1)^{-1} \leq M$.

Let $X=\mathbf{R}^{n} \times \mathbf{R}_{-}$, and let $a(t)$ be a positive continuous function defined on $(-\infty, 0]$. We shall consider the following sets,

$$
\begin{aligned}
H & =H(X)=\left\{u \in W_{\mathrm{loc}}^{2 ; 1, n+1}(X) \mid L u=0\right\}, \\
H_{+} & =\{u \in H \mid u \geq 0\}, \\
H_{a} & =\left\{u \in H_{+} \mid \int_{-\infty}^{0} u(0, t) a(t) d t \leq 1\right\}, \\
H_{a}^{1} & =\left\{u \in H_{a} \mid \int_{-\infty}^{0} u(0, t) a(t) d t=1\right\} .
\end{aligned}
$$


It follows from Theorem 1.3 and from known a priori estimates $[\mathbf{5}, \mathbf{6}]$ that $H_{+}$ is a closed convex cone in the locally convex linear space $H$, in the topology of uniform convergence on compact sets. Moreover $H_{a}$ is a metrizable cap in $H_{+}$(i.e. $H_{a}$ is a compact convex set and $H_{+} \backslash H_{a}$ is convex). It is easy to see that $H_{+}$is well capped.

Recall that $H_{+}$is a harmonic space in the sense of Bauer, this implies that $H_{a}$ is a simplex.

Let $\mathscr{C}(K)$ be a convex cone (set), we shall denote by $\operatorname{ex} r \mathscr{C}(\operatorname{ex} K)$ the set of all extreme rays (points) of $\mathscr{C}(K)$.

Since $H_{+}$is a proper cone, we have

$$
\operatorname{ex} H_{a}=\{0\} \cup\left\{\operatorname{ex} r H_{+} \cap H_{a}^{1}\right\} .
$$

2. Minimal solutions for the time independent case. Suppose that $L$ is a second order parabolic operator on $X$ of the form (1.1), which satisfies (1.2) and (1.3). Suppose further that the coefficients of $L$ are time independent, that is

$$
L u=u_{t}+P\left(x, \partial_{x}\right) u,
$$

where $P$ is a uniformly elliptic operator on $\mathbf{R}^{n}$.

We shall denote by $\mathscr{K}_{P+\lambda}\left(\mathbf{R}^{n}\right)$ the set of all positive strong solutions of the equation $(P+\lambda) u=0$ in $\mathbf{R}^{n}$ s.t. $u(0)=1$.

The following theorem characterizes ex $r H_{+}$.

THEOREM 2.1. Let $L u=u_{t}+P(x, \partial x) u$. Then $u \in \operatorname{ex} H_{a} \backslash\{0\}$ iff

$$
u(x, t)=\left(C_{a}(\lambda)\right)^{-1} e^{\lambda t} u_{\lambda}(x),
$$

where $u_{\lambda} \in \operatorname{ex} \mathscr{K}_{P+\lambda}\left(\mathbf{R}^{n}\right), C_{a}(\lambda)=\int_{-\infty}^{0} e^{\lambda t} a(t) d t$ and $\lambda \geq \lambda^{*}$ for some $\lambda^{*} \in \mathbf{R}$.

REMARK 2.2. The first part of Theorem 2.1 was proved independently also by A. Koranyi and J. C. Taylor [4].

Proof of ThEOREM 2.1. Let $u \in \operatorname{ex} H_{a} \backslash\{0\}$, and let $0<\delta<4$. Consider the function

$$
v_{\delta}(x, t)=C^{-1} u(x, t-\delta) .
$$

Since the coefficients of $L$ are time independent, $v_{\delta} \in H_{+}$. Moreover, using Harnack's inequality in $Q\left(x, t-2 \delta, \delta^{1 / 2}, 2\right)$ we obtain

$$
v_{\delta}(x, t)=C^{-1} u(x, t-\delta) \leq u(x, t) \text { for all } t<0 .
$$

Hence the function $w_{\delta}(x, t)=u(x, t)-v_{\delta}(x, t)$ is also in $H_{+}$. From (2.4) we deduce that

$$
k_{\delta}=\int_{-\infty}^{0} v_{\delta}(0, t) a(t) d t \leq 1,
$$

therefore $v_{\delta}, w_{\delta} \in H_{a}$. Suppose that $0<k_{\delta}<1$. Consequently we have

$$
u(x, t)=k_{\delta}\left(k_{\delta}^{-1} v_{\delta}(x, t)\right)+\left(1-k_{\delta}\right)\left(\left(1-k_{\delta}\right)^{-1} w_{\delta}(x, t)\right) .
$$

Since $u \in \operatorname{ex} H_{\alpha}$ it follows from (2.4) or (2.6) that

$$
v_{\delta}(x, t)=C^{-1} u(x, t-\delta)=\nu_{\delta} u(x, t),
$$

where $\nu_{\delta}$ is a nonnegative constant. 
If $\nu_{\delta}>0$ then $u(x, t)=C_{\delta} e^{\lambda t} \varphi_{\delta}(x, t)$ where $\varphi_{\delta}(x, t)$ is a periodic function in $t$ with period $\delta$. If $\nu_{\delta}=0$ it follows that $u(x, t)=0$ for all $x \in \mathbf{R}^{n}$ and $t<-\delta$. Since $\delta>0$ is arbitrary small and $u \neq \equiv 0$ it follows that

$$
u(x, t)=\left(C_{a}(\lambda)\right)^{-1} e^{\lambda t} u_{\lambda}(x),
$$

where $u_{\lambda} \in \mathscr{K}_{P+\lambda}\left(\mathbf{R}^{n}\right)$. By (1.10) $u \in H_{a}^{1}$, therefore $C_{a}(\lambda)=\int_{-\infty}^{0} e^{\lambda t} a(t) d t$. Using Harnack's inequality we have $\lambda>\lambda^{*}$ for some $\lambda^{*}$. It is also easy to check that $u_{\lambda} \in \operatorname{ex} \mathscr{K}_{P+\lambda}\left(\mathbf{R}^{n}\right)$.

Suppose now that $u_{0} \in H_{a}$ is given by (2.2), i.e.

$$
u_{0}(x, t)=\left(C_{a}\left(\lambda_{0}\right)\right)^{-1} e^{\lambda_{0} t} u_{\lambda_{0}}(x),
$$

where $u_{\lambda_{0}} \in \operatorname{ex} \mathscr{K}_{P+\lambda_{0}}\left(\mathbf{R}^{n}\right)$. We shall show that $u_{0} \in \operatorname{ex} H_{a}$. Consider the sets

$$
E_{a}=\{0\} \cup\left\{u \in H_{a}^{1} \mid u(x, t)=\left(C_{a}(\lambda)\right)^{-1} e^{\lambda t} u_{\lambda}(x), u_{\lambda} \in \mathscr{K}_{P+\lambda}\left(\mathbf{R}^{n}\right), \lambda \geq \lambda^{*}\right\}
$$

$$
E_{\lambda_{0}}^{a}=\left\{u \in E_{a} \mid u(x, t)=\left(C_{a}\left(\lambda_{0}\right)\right)^{-1} e^{\lambda_{0} t} u_{\lambda_{0}}(x)\right\} .
$$

It is clear that $E_{\lambda_{0}}^{a}$ is a compact set in $H_{a}$. We shall show that $E_{a}$ is also compact in $H_{a}$.

Suppose that $\left\{u_{k}\right\}_{k=1}^{\infty} \subset E_{a}, u_{k} \in E_{\lambda_{k}}^{a}$ and $u_{k} \underset{k \rightarrow \infty}{\longrightarrow} u \in H_{a}$. There exists a subsequence $\left\{\lambda_{k_{l}}\right\}_{l=1}^{\infty}$ s.t. $\lambda_{k_{l}} \underset{l \rightarrow \infty}{\longrightarrow} \tilde{\lambda} \in \mathbf{R}$ or $\lambda_{k_{l}} \underset{l \rightarrow \infty}{\longrightarrow} \infty$.

Notice that if $\lambda>0$ then for every $t<0$ we have

$$
C_{a}(\lambda) \geq \int_{t / 2}^{0} e^{\lambda \tau} a(\tau) d \tau \geq e^{\lambda t / 2} \int_{t / 2}^{0} a(\tau) d \tau=c(t) e^{\lambda t / 2} .
$$

Hence, if $\lambda_{k_{l}} \underset{l \rightarrow \infty}{\longrightarrow} \infty$, then $\exp \left(\lambda_{k_{l}} t\right)\left(C_{a}\left(\lambda_{k_{l}}\right)\right)^{-1} \underset{l \rightarrow \infty}{\longrightarrow} 0$ for all $t<0$. Therefore $u(0, t)=0$ for all $t<0$, and by the Harnack inequality $u \equiv 0$.

If $\lambda_{k_{l}} \underset{l \rightarrow \infty}{\longrightarrow} \tilde{\lambda}$, then there exists a subsequence of $\left\{u_{\lambda_{k}}(x)\right\}_{k=1}^{\infty}$ that convergence to $u_{\tilde{\lambda}} \in \mathscr{K}_{P_{+} \tilde{\lambda}}^{\infty}\left(\mathbf{R}^{n}\right)$. Using the dominated (monotone) convergence theorem we see that

$$
C_{a}\left(\lambda_{k_{l}}\right) \underset{l \rightarrow \infty}{\longrightarrow} C_{a}(\tilde{\lambda})
$$

Hence

$$
u(x, t)=\left(C_{a}(\tilde{\lambda})\right)^{-1} e^{\tilde{\lambda} t} u_{\tilde{\lambda}}(x) \in E_{a}
$$

and $E_{a}$ is a compact set.

Recall that $H_{a}$ is a simplex and ex $H_{a} \subseteq E_{a}$, therefore there exists a unique probability measure $\mu$ on $E_{a}$ s.t. $\mu\left(\operatorname{ex} H_{a}\right)=1$ and

$$
u_{0}(x, t)=C_{a}\left(\lambda_{0}\right)^{-1} e^{\lambda_{0} t} u_{\lambda_{0}}(x)=\int_{E_{a}} v(x, t) d \mu(v) .
$$

Substitute $x=0$ in (2.13) we obtain

$$
\left(C_{a}\left(\lambda_{0}\right)\right)^{-1} e^{\lambda_{0} t}=\int_{E_{a}}\left(C_{a}(\lambda(v))\right)^{-1} e^{\lambda(v) t} d \mu(v) .
$$


Since $\lambda<\lambda_{0}$ implies $\lim _{t \rightarrow-\infty} e^{\left(\lambda-\lambda_{0}\right) t}=\infty$ we find that $\mu\left(\bigcup_{\lambda<\lambda_{0}} E_{\lambda}^{a}\right)=0$. It is also clear that $\mu(\{0\})=0$. The left-hand side of (2.14) is well defined also for $t=0$, this implies that

$$
\left(C_{a}\left(\lambda_{0}\right)\right)^{-1}=\int_{\left\{v \in E_{\lambda}^{a} \mid \lambda \geq \lambda_{0}\right\}}\left(C_{a}(\lambda(v))\right)^{-1} d \mu(v) .
$$

Since $\left(C_{a}(\lambda)\right)^{-1}$ is an increasing function of $\lambda$ it follows from (2.15) that $\operatorname{supp}(\mu)$ $\subseteq E_{\lambda_{0}}^{a}$ and we have

$$
u_{\lambda_{0}}(x)=\int_{E_{\lambda_{0}}^{a}} v_{\lambda_{0}}(x) d \mu\left(v_{\lambda_{0}}\right), \quad x \in \mathbf{R}^{n} .
$$

Since $E_{\lambda_{0}}^{a}$ is homeomorphic to a closed set in $\mathscr{K}_{P+\lambda_{0}}\left(\mathbf{R}^{n}\right)$ and

$$
u_{\lambda_{0}}(x) \in \operatorname{ex} \mathscr{K}_{P+\lambda_{0}}\left(\mathbf{R}^{n}\right)
$$

we conclude that $\mu=\delta_{u_{0}}$ and $u_{0} \in \operatorname{ex} H_{a}$.

REMARK 2.3. We can use Theorem 2.1 and the representation theorems in [1] and [7] to derive representation theorems for nonnegative solutions of the equation $L u=u_{t}+P(x, \partial) u=0$ in $\mathbf{R}^{n} \times R_{-}$where $P$ has periodic coefficients, or $P$ is a Schrödinger operator with a principally radially symmetric potential. The periodic case can also be considered as a special case of the result of the next section.

3. The representation theorem for the periodic case. In this section we shall consider a second order parabolic operator $L$ of the form (1.1). We assume that $L$ satisfies (1.2) and (1.3). Moreover we assume that

$$
a_{i j} \in C^{2+\alpha ; 1+\alpha / 2}(X), \quad b_{i} \in C^{1+\alpha ; a / 2}(X), \quad c \in C^{\alpha ; \alpha / 2}(X), \quad 1 \leq i, j \leq n,
$$

where $0<\alpha \leq 1$. Finally we shall assume that the coefficients of $L$ are periodic in $x_{1}, \ldots, x_{n}$, and $t$ with period 1 .

THEOREM 3.1. Suppose that L satisfies the above assumptions. Denote

$$
C_{a}(u)=\int_{-\infty}^{0} u(0, t) a(t) d t .
$$

Then $u \in \operatorname{ex} H_{a} \backslash\{0\}$ iff $u$ is of the form:

$$
u(x, t)=\left(C_{a}(u)\right)^{-1} \exp (\xi \cdot x+\lambda t) \varphi_{(\xi, \lambda)}(x, t),
$$

where $\xi \in \mathbf{R}^{n}, \lambda \geq \lambda^{*}$ for some $\lambda^{*} \in \mathbf{R}$, and $\varphi(\xi, \lambda)$ is a positive periodic function in $x_{1}, \ldots, x_{n}$ and $t$ with period 1 .

DEFINITION 3.2. $u \in H_{+} \backslash\{0\}$ is said to be an exponential solution with exponent $(\xi, \lambda) \in \mathbf{R}^{n} \times \mathbf{R}$, if $u$ is a scalar multiple of a solution of the form (3.3).

REMARK 3.3. Using Harnack's inequality it is easy to see that two exponential solutions with the same exponent are linearly dependent. We shall denote an exponential solution with exponent $(\xi, \lambda)$ and $\varphi_{(\xi, \lambda)}(0,-1)=1$ by $u_{(\xi, \lambda)}(x, t)$. Let

$$
\begin{aligned}
& E=\{0\} \cup\left\{u \in H_{+} \mid u \text { is an exponential solution }\right\}, \\
& \Gamma=\left\{(\xi, \lambda) \in \mathbf{R}^{n} \times \mathbf{R} \mid u_{(\xi, \lambda)} \in E\right\}
\end{aligned}
$$

The following theorem shows that $\Gamma$ is of a paraboloid shape. 
THEOREM 3.4. $\Gamma$ is a strictly convex $n$ dimensional analytic manifold in $\mathbf{R}^{n} \times$ R. Moreover if $\lambda_{1}<\lambda_{2}$ then $\Gamma^{\lambda_{1}} \subset \subset \Gamma^{\lambda_{2}}$, where

$$
\Gamma^{\lambda}=\operatorname{conv}\left\{\xi \in \mathbf{R}^{n} \mid(\xi, \lambda) \in \Gamma\right\} .
$$

PROOF OF THEOREMS 3.1 AND 3.4. As in the proof of Theorem 2.1 we show that if $u \in \operatorname{ex} H_{a} \backslash\{0\}$, then

$$
u(x, t-1)=\nu u(x, t) \text { for all }(x, t) \in X,
$$

where $\nu \geq 0$. From the uniqueness of the positive Cauchy problem [2, 3], it follows that $\nu>0$. Hence

$$
u(x, t)=e^{\lambda t} \psi(x, t)
$$

where $\lambda \in \mathbf{R}$ and $\psi(x, t)$ is a nonnegative periodic function in $t$ with period 1 .

Let $e_{i}, i=1, \ldots, n$, denote the unit vector in the direction of $x_{i}$ in $\mathbf{R}^{n}$. Consider the functions

$$
u_{i}(x, t)=e^{-\lambda} C^{-1} u\left(x+e_{i}, t\right), \quad i=1, \ldots, n .
$$

From (3.7) and Harnack's inequality it follows that

$$
u_{i}(x, t)=C^{-1} u\left(x+e_{i}, t-1\right) \leq u(x, t),
$$

hence $u_{i} \in H_{a}$. Since $u \in \operatorname{ex} H_{a}$ it follows that

$$
u_{i}(x, t)=\nu_{i} u(x, t), \quad i=1, \ldots, n,
$$

for some $\nu_{i}>0$. Consequently $u$ is an exponential solution. Using Harnack's inequality it is easy to see that $\lambda>\lambda^{*}$ for some fixed $\lambda^{*} \in \mathbf{R}$ and that $\varphi_{(\xi, \lambda)}(x, t)>$ 0 . We can assume that $\varphi_{(\xi, \lambda)}(0,-1)=1$. Using Harnack's inequality once again we obtain

$$
C^{-1} \min \left(1, e^{-\lambda}\right) \leq \varphi_{(\xi, \lambda)}(0, t) \leq C \max \left(1, e^{\lambda}\right) .
$$

It follows from (3.11) that there exists a function $\tilde{a}: \mathbf{R}_{-} \rightarrow \mathbf{R}_{+}$s.t. $E / \mathbf{R}_{+} \subseteq H_{\tilde{a}}$. Let $E_{a}=\{0\} \cup E \cap H_{a}^{1}$, and define

$$
\Gamma_{a}=\left\{(\xi, \lambda) \in \Gamma \mid u_{(\xi, \lambda)} \in E_{a}\right\} .
$$

It is easy to see that $E_{\tilde{a}}$ is a closed set and that $\{\infty\} \cup \Gamma \cong E_{\tilde{a}}$. Let $u \in H_{\tilde{a}}$, from the Krein-Milman theorem it follows that there exists a probability measure $\mu$ on $\Gamma \cup\{\infty\}$ s.t.

$$
u(x, t)=C_{\tilde{a}}(u) \int_{\Gamma \cup\{\infty\}}\left(C_{\tilde{a}}\left(u_{(\xi, \lambda)}\right)\right)^{-1} \exp (\xi \cdot x+\lambda t) \varphi_{(\xi, \lambda)}(x, t) d \mu(\xi, \lambda) .
$$

Suppose now that $u=u_{\left(\xi_{0}, \lambda_{0}\right)}$ for some $\left(\xi_{0}, \lambda_{0}\right) \in \Gamma$, then for all $(m, \tau) \in$ $\mathbf{Z}^{n} \times \mathbf{Z}_{-}$we have

$$
\exp \left(\xi_{0} \cdot m+\lambda_{0} \cdot \tau\right)=C_{\tilde{a}}\left(u_{\left(\xi_{0}, \lambda_{0}\right)}\right) \int_{\Gamma \cup\{\infty\}}\left(C_{\tilde{a}}\left(u_{(\xi, \lambda)}\right)\right)^{-1} \exp (\xi \cdot m+\lambda \tau) d \mu(\xi, \lambda) .
$$

Hence

$$
\int_{\Gamma \cup\{\infty\}} \exp (\xi \cdot m+\lambda \tau) d \nu=0 \quad \text { for all }(m, \tau) \in \mathbf{Z}^{n} \times \mathbf{Z}_{-}
$$


where

$$
d \nu=\left(C_{\tilde{a}}\left(u_{(\xi, \lambda)}\right)\right)^{-1} d \mu(\xi, \lambda)-\left(C_{\tilde{a}}\left(u_{\left(\xi_{0}, \lambda_{0}\right)}\right)\right)^{-1} \delta_{\left(\xi_{0}, \lambda_{0}\right)}(\xi, \lambda)
$$

is a signed measure.

Notice that $\mathscr{A}=\operatorname{span}\{\exp (\xi \cdot m+\lambda \tau)\}_{(m, \tau) \in \mathbf{Z}^{n} \times \mathbf{Z}_{-}}$is an algebra of real continuous functions on the compact space $\Gamma \cup\{\infty\}$ which separates the points of $\Gamma \cup\{\infty\}$ and does not vanish at any point. From the Stone-Weierstrass theorem it follows that $\overline{\mathscr{A}}=C(\Gamma \cup\{\infty\})$. Consequently (3.15) and the Riesz representation theorem imply now that $\nu=0$, hence $u_{\left(\xi_{0}, \lambda_{0}\right)} \in \operatorname{ex} H_{\tilde{a}}$ for all $\left(\xi_{0}, \lambda_{0}\right) \in \Gamma$. The last argument also gives a proof for the uniqueness of the integral representation (3.13).

We shall show now that $\Gamma=\operatorname{ex}(\operatorname{conv} \Gamma)$. Suppose that $(\xi, \lambda) \in \Gamma \backslash \operatorname{ex}(\operatorname{conv} \Gamma)$, then by the Carathéodory theorem there exist $\left(\xi_{i}, \lambda_{i}\right) \in \operatorname{ex}(\operatorname{conv}(\Gamma))$ and $l_{i} \geq 0$, $0 \leq i \leq n+1$ s.t.

$$
(\xi, \lambda)=\sum_{i=0}^{n+1} l_{i}\left(\xi_{i}, \lambda_{i}\right), \quad \sum_{i=0}^{n+1} l_{i}=1 .
$$

It follows that for all $(x, t) \in X$

$$
\xi \cdot x+\lambda t \leq \max _{0 \leq i \leq n+1}\left(\xi_{i} \cdot x+\lambda_{i} t\right) .
$$

Let $v(x, t)=\sum_{i=0}^{n+1} u_{\left(\xi_{i}, \lambda_{i}\right)}(x, t)$. Using (3.17) and the Harnack inequality we obtain

$$
u_{(\xi, \lambda)}(x, t) \leq C v(x, t) \text { for all }(x, t) \in X,
$$

where $C$ is a positive constant. Define

$$
w(x, t)=\alpha^{-1}\left(C v(x, t)-u_{(\xi, \lambda)}(x, t)\right),
$$

where

$$
\alpha^{-1}=\int_{-\infty}^{0}\left(C v(0, t)-u_{(\xi, \lambda)}(0, t)\right) a(t) d t .
$$

Let $\mu$ be the probability measure on $\Gamma \cup\{\infty\}$ s.t.

$$
w(x, t)=\int_{\Gamma \cup\{\infty\}} u_{(\tilde{\xi}, \tilde{\lambda})}(x, t) d \mu(\tilde{\xi}, \tilde{\lambda}) .
$$

Notice that

$$
v(x, t)=\alpha C^{-1} w(x, t)+C^{-1} u_{(\xi, \lambda)}(x, t) .
$$

(3.19) and the uniqueness of the integral representation imply that

$$
\sum_{i=0}^{n+1} \delta_{\left(\xi_{i}, \lambda_{i}\right)}=\alpha C^{-1} \mu+C^{-1} \delta_{(\xi, \lambda)}
$$

This yields a contradiction which shows that $\Gamma=\operatorname{ex}(\operatorname{conv} \Gamma)$. By the same way one can show that $\xi \in \Gamma^{\lambda_{1}}$ implies $\xi \notin e x \Gamma^{\lambda_{2}}$ for all $\lambda_{2}>\lambda_{1}$.

We shall show that $\Gamma$ is an $n$ dimensional analytic manifold. For this purpose let us introduce the following Banach spaces.

$$
\mathscr{D}=\left\{\begin{array}{l|l}
u \in C^{2+\alpha ; 1+\alpha / 2}(X) & \begin{array}{l}
u \text { is a periodic function in } x_{1}, \ldots, x_{n} \text { and } \\
t \text { with period } 1 .
\end{array}
\end{array}\right\},
$$




$$
\mathscr{F}=\left\{\begin{array}{l|l}
f \in C^{\alpha ; \alpha / 2}(X) & \begin{array}{l}
f \text { is a periodic function in } x_{1}, \ldots, x_{n} \text { and } \\
t \text { with period 1. }
\end{array}
\end{array}\right\} .
$$

It is clear that $\mathscr{D}$ is compactly imbedded in $\mathscr{F}$. Consider the family of the operators $L_{(\xi, \lambda)}: \mathscr{D} \rightarrow \mathscr{F},(\xi, \lambda) \in \mathbf{R}^{n} \times \mathbf{R}$,

$$
L_{(\xi, \lambda)}=\exp (-\xi \cdot x-\lambda t) L \exp (\xi \cdot x+\lambda t) .
$$

Using the definitions of $L_{(\xi, \lambda)}$ and $\Gamma$ we see that the equation

$$
L_{(\xi, \lambda)} u=0
$$

admits a positive solution in $\mathscr{D}$ iff $(\xi, \lambda) \in \Gamma$. It is known (see for example [11, p. 76]) that $L_{(\xi, \lambda)}+s I$ is an invertible operator if $s$ is large enough, and the inverse operator is positive and compact. Therefore the operator $L_{(\xi, \lambda)}$ has a positive eigenfunction with a simple real eigenvalue $\Lambda(\xi, \lambda)$. It is easy to check that

$$
\Gamma=\left\{(\xi, \lambda) \in \mathbf{R}^{n} \times \mathbf{R} \mid \Lambda(\xi, \lambda)=0\right\} .
$$

The set $\left\{L_{(\xi, \lambda)} \mid(\xi, \lambda) \in \mathbf{R}^{n} \times \mathbf{R}\right\}$ is an analytic family of operators, hence $\Lambda(\xi, \lambda)=\Lambda\left(\xi_{1}, \ldots, \xi_{n}, \lambda\right)$ is an analytic function. Consider the functions $\widetilde{\Lambda}(\xi)=$ $\Lambda(\xi, 0)$ and $\psi(\xi, \lambda)=\tilde{\Lambda}(\xi)+\lambda$. Then $\psi(\xi, \lambda)$ is also an analytic function and $\operatorname{grad} \psi \neq 0$. Moreover

$$
\Gamma=\left\{(\xi, \lambda) \in \mathbf{R}^{n} \times \mathbf{R} \mid \psi(\xi, \lambda)=0\right\} .
$$

Therefore $\Gamma$ is an $n$ dimensional analytic manifold. From the first part of the proof it follows now that $\Gamma$ is the boundary of a strictly convex set in $\mathbf{R}^{n} \times \mathbf{R}$. Since we have also shown that $\xi \in \Gamma^{\lambda_{1}}$ implies $\xi \notin \operatorname{ex} \Gamma^{\lambda_{2}}$ for all $\lambda_{2}>\lambda_{1}$, it follows that $\Gamma^{\lambda_{1}} \subset \subset \Gamma^{\lambda_{2}}$ for all $\lambda_{1}<\lambda_{2}$.

In the last proof we have shown

REPRESENTATION THEOREM. Suppose that $u \in H_{a}^{1}$ for some positive function $a: \mathbf{R}_{-} \rightarrow \mathbf{R}_{+}$. Then there exists a unique probability measure on $\Gamma_{a} \cup\{\infty\}$ s.t.

$$
u(x, t)=\int_{\Gamma_{a} \cup\{\infty\}}\left(C_{a}\left(u_{(\xi, \lambda)}\right)\right)^{-1} u_{(\xi, \lambda)}(x, t) d \mu(\xi, \lambda),
$$

where $u_{(\xi, \lambda)}$ is the exponential solution of the equation $L u=0$ in $X$ with exponent $(\xi, \lambda)$ s.t. $\varphi_{(\xi, \lambda)}(0,-1)=1$.

REMARK 3.5. One can derive analogue results to the results of this paper for the nonnegative solutions of $L u=0$ in $\mathbf{R}^{n} \times \mathbf{R}$. The proofs differ only in minor details from the proofs for $\mathbf{R}^{n} \times \mathbf{R}_{-}$.

\section{REFERENCES}

1. S. Agmon, On positive solutions of elliptic equations with periodic coefficients in $\mathbf{R}^{n}$, spectral results and extensions to elliptic operators on Riemannian manifolds, Proc. Internat. Conf. on Differential Equations (I. W. Knowles and R. T. Lewis, eds.), North-Holland Math. Studies, No. 92, 1984, pp. 7--17.

2. D. G. Aronson, Non-negative solutions of linear parabolic equations, Ann. Scuola Norm. Sup. Pisa (3) 22 (1968), 607-694.

3. A. Friedman, On the uniqueness of the Cauchy problem for parabolic equations, Amer. J. Math. 81 (1959), 503-511. 
4. A. Koranyi and J. C. Taylor, Minimal solutions of the heat equation and uniqueness of the positive Cauchy problem on homogeneous spaces, Proc. Amer. Math. Soc. 94 (1985), 273-278.

5. N. V. Krylov and M. V. Safanov, A certain property of solutions of parabolic equations with measurable coefficients, Math. USSR Izv. 16 (1981), 151-164.

6. O. A. Ladyženskaja, V. A. Solonnikov and N. N. Ural'ceva, Linear and quasilinear equations of parabolic type, Transl. Math. Monographs, vol. 23, Amer. Math. Soc., Providence, R.I., 1968.

7. M. Murata, Structure of positive solutions to $(-\Delta+V) u=0$ in $\mathbf{R}^{n}$, Duke Math. J. 53 (1986), 869-943.

8. Y. Pinchover, Sur les solutions positives d'équations elliptiques et paraboliques dans $\mathbf{R}^{n}$, C. R. Acad. Sci. I Paris, 302 (1986), 447-450.

9. __ Positive solutions of second order elliptic equations, Ph.D. Thesis, Hebrew Univ. of Jerusalem, Jerusalem, 1986. (Hebrew)

10. D. V. Widder, Positive temperature on an infinite rod, Trans. Amer. Math. Soc. 55 (1944), 85-95.

11. V. V. Zhikov, Asymptotic behavior and stabilization of solutions of second order parabolic equations with lower order terms, Trans. Moscow Math. Soc. 2 (1984), 69-99.

Department of Mathematics, University of California, Los Angeles, CaliFORNIA 90024

Current address: Department of Mathematics, Technion-Israel Institute of Technology, 32000 Haifa, Israel 